\title{
The CNDP1 (CTG) 5 Polymorphism Is Associated with Biopsy-Proven Diabetic Nephropathy, Time on Hemodialysis, and Diabetes Duration
}

\author{
Thomas Albrecht, ${ }^{1}$ Shiqi Zhang, ${ }^{1,2}$ Jana D. Braun, ${ }^{1}$ Li Xia, ${ }^{1,2}$ Angelica Rodriquez, \\ Jiedong Qiu, ${ }^{1}$ Verena Peters, ${ }^{3}$ Claus P. Schmitt, ${ }^{3}$ Jacob van den Born, ${ }^{4}$ Stephan J. L. Bakker, \\ Alexander Lammert, ${ }^{1}$ Hannes Köppel, ${ }^{1}$ Peter Schnuelle, ${ }^{1}$ Bernhard K. Krämer, ${ }^{1}$ \\ Benito A. Yard, ${ }^{1}$ and Sibylle J. Hauske ${ }^{1}$ \\ ${ }^{1}$ Fifth Medical Department (Nephrology/Endocrinology/Rheumatology), University Medical Center Mannheim, \\ University of Heidelberg, Mannheim, Germany \\ ${ }^{2}$ Department of Endocrinology, The First Affiliated Hospital of Anhui Medical University, Hefei, China \\ ${ }^{3}$ Centre for Pediatric and Adolescent Medicine, University of Heidelberg, Heidelberg, Germany \\ ${ }^{4}$ Nephrology, University Medical Center Groningen, University of Groningen, Groningen, Netherlands \\ Correspondence should be addressed to Thomas Albrecht; t.albrecht@stud.uni-heidelberg.de
}

Received 1 December 2016; Revised 23 February 2017; Accepted 13 March 2017; Published 3 May 2017

Academic Editor: Feng Wang

Copyright (C) 2017 Thomas Albrecht et al. This is an open access article distributed under the Creative Commons Attribution License, which permits unrestricted use, distribution, and reproduction in any medium, provided the original work is properly cited.

\begin{abstract}
Considering that the homozygous CNDP1 $(\mathrm{CTG})_{5}$ genotype affords protection against diabetic nephropathy (DN) in female patients with type 2 diabetes, this study assessed if this association remains gender-specific when applying clinical inclusion criteria (CIC-DN) or biopsy proof (BP-DN). Additionally, it assessed if the prevalence of the protective genotype changes with diabetes duration and time on hemodialysis and if this occurs in association with serum carnosinase (CN-1) activity. Whereas the distribution of the $(\mathrm{CTG})_{5}$ homozygous genotype in the no-DN and CIC-DN patients was comparable, a lower frequency was found in the BP-DN patients, particularly in females. We observed a significant trend towards high frequencies of the $(\mathrm{CTG})_{5}$ homozygous genotype with increased time on dialysis. This was also observed for diabetes duration but only reached significance when both $(\mathrm{CTG})_{5}$ homo- and heterozygous patients were included. $\mathrm{CN}-1$ activity negatively correlated with time on hemodialysis and was lower in $(\mathrm{CTG})_{5}$ homozygous patients. The latter remained significant in female subjects after gender stratification. We confirm the association between the CNDP1 genotype and DN to be likely gender-specific. Although our data also suggest that $(\mathrm{CTG})_{5}$ homozygous patients may have a survival advantage on dialysis and in diabetes, this hypothesis needs to be confirmed in a prospective cohort study.
\end{abstract}

\section{Introduction}

Diabetic nephropathy (DN) occurs in approximately $40 \%$ of patients with type 1 and type 2 diabetes [1] and is the leading cause of end-stage renal disease (ESRD) [2]. Compelling evidence has shown that susceptibility to $\mathrm{DN}$ is genetically determined $[3,4]$. Amongst the reported linkage studies, there seems to be consistency in the linkage between human chromosome 18q22.3-q23 and DN [4-7]; linkage to the DN trait on chromosomes 7q21.3, 10p15.3, and 14q23.1 has also been reported [7]. Linkage with 18q22.3 was observed in populations of different ethnicities, for example, American Indians [8], Afro-Americans [9], and Caucasians [5].

Janssen et al. initially postulated that the CNDP1 gene on chromosome 18q22.3-q23, encoding serum carnosinase $(\mathrm{CN}-1)$, is a susceptibility gene for $\mathrm{DN}$ in type 2 diabetes mellitus (T2DM) patients [10]. It was found that T2DM patients homozygous for the CNDP1 (CTG) ${ }_{5}$ allele are less 
frequently affected by DN compared to T2DM patients with other CNDP1 genotypes [10]. The prevalence of the $(\mathrm{CTG})_{5}$ allele strongly varies with different ethnicities. While homozygosity for the $(\mathrm{CTG})_{5}$ allele is more frequent in the European population ( $38.6 \%$ in healthy controls and $29.3 \%$ in diabetic patients with ESRD) [11], this genotype seems to be much more rare in the Asian population with a high prevalence of $\mathrm{DN}[12,13]$. It has also been reported that the association between the CNDP1 genotype and DN is sexspecific and independent of susceptibility to T2DM [14].

Since most patients with T2DM are not formally evaluated with a renal biopsy, the diagnosis of $\mathrm{DN}$ is based on clinical criteria, for example, persistent macroalbuminuria on at least 2 independent occasions (albumin excretion rate $>300 \mathrm{mg} / \mathrm{d}$ or $>200 \mathrm{mg} / \mathrm{l}$ or ACR (albumin/creatinine ratio) $>300 \mathrm{mg} / \mathrm{g}$ ). Yet, biopsy-based retrospective evaluations of the prevalence of nondiabetic renal disease (NDRD) in T2DM patients revealed a high percentage of patients having NDRD without evidence of concurrent DN $[15,16]$. Although the predictive value of clinical criteria for DN in T2DM patients can be improved by the presence of proliferative retinopathy $[17,18]$, genetic studies that use clinical inclusion criteria (CIC) for group allocation still bear the risk of wrongly assigning patients to the DN group. In the present study, we assessed if the association between CNDP1 and DN is still observed when applying CIC or biopsy-proven diabetic nephropathy (BP-DN) and, if so, whether this is only observed in female T2DM patients. Since also an association between the CNDP1 (CTG) ${ }_{5}$ homozygous genotype and cardiovascular mortality has been reported to be sex-specific [19], we also assessed if the prevalence of this genotype changes with diabetes duration and time on dialysis. Since T2DM patients on dialysis have a high mortality risk, it would be expected that the proportion of CNDP1 (CTG) homozygous patients would decrease with time on dialysis, particularly in females.

\section{Materials and Methods}

2.1. Patients. Patients were recruited between 2011 and 2014 from the Fifth Medical Clinic and Dialysis Unit at the University Medical Centre Mannheim and different nephrology practices in proximity (Centre for Renal Disease, Weinheim, Lindenfels, Viernheim; Nephrocare Ludwigshafen GmbH; KfH Nierenzentrum, Ludwigshafen; Nephrology Practice Frankenthal, Bad Dürkheim, Lampertheim). After screening of clinical records $(n=384)$ and patients with a biopsyproven renal diagnosis $(n=52)$, a total of 436 patients were deemed to be eligible for this study. Due to the retrospective nature of this study, indications for renal biopsies were not uniform and the histological evaluation was undertaken by different pathologists. Out of the 436 initially selected patients, 66 were excluded because of incomplete clinical data or missing informed consent. The remaining patients $(n=370)$ were allocated to 5 different groups, that is, 130 T2DM patients without DN (no-DN), 108 T2DM patients with CIC-DN, 30 T2DM patients with BP-DN, 80 patients with CIC nondiabetic renal disease (CIC-NDRD), and 22 patients with biopsy-proven nondiabetic renal disease
(BP-NDRD) (Figure 1). Patients without renal biopsy material were allocated on the basis of clinical criteria as specified below, whereas patients with renal biopsy material were allocated either to the BP-DN or to the BP-NDRD group. The ethnicity distributions in the no-DN and BP-DN groups were broadly comparable (no-DN: 75\% from Germany, 12.5\% from Turkey; BP-DN: 70\% from Germany, $17 \%$ from Turkey).

Inclusion criteria for clinical diagnosis of $\mathrm{DN}$ were as follows: persistent macroalbuminuria on at least 2 independent occasions (albumin excretion rate $>300 \mathrm{mg} / \mathrm{d}$ or $>200 \mathrm{mg} / \mathrm{l}$ or ACR (albumin/creatinine ratio) $>300 \mathrm{mg} / \mathrm{g}$ ) in combination with the diagnosis of diabetic retinopathy (DR) (all severity degrees were allowed). This combination was obligatory to reduce the possibility that cases with proteinuria due to renal disease other than DN (NDRD) were included [20]. Anuric patients with a history of macroalbuminuria were included. Exclusion criteria were urinary tract infection or fever at the time of urine investigation, documented renal disease other than $\mathrm{DN}$, and a history of kidney transplantation.

T2DM patients without DN (=control group) fulfilled the following criteria: diabetes duration of at least 15 years accompanied by normoalbuminuria on at least two independent occasions (albumin excretion rate $<30 \mathrm{mg} / \mathrm{d}$ or $<20 \mathrm{mg} / \mathrm{l}$ or ACR $<30 \mathrm{mg} / \mathrm{g}$ ). Since approximately $80 \%$ of the diabetic patients in this group were on ACE inhibitors or $\mathrm{AT}_{1}$ blockers, false-negative results based on albuminuria could not be excluded. However, to minimize the possibility of DN patients in the control group, only the normoalbuminuric patients with no or mild nonproliferate $\mathrm{DR}$ were included since the presence of nephropathy without significant DR is rare $[18,21,22]$. Diabetes mellitus was defined by a documented history of diabetes or a fasting blood glucose of $\geq 7.0 \mathrm{mmol} / \mathrm{l}(126 \mathrm{mg} / \mathrm{dl})$, a casual plasma glucose level of $\geq 11.1 \mathrm{mmol} / \mathrm{l}(200 \mathrm{mg} / \mathrm{dl})$, or a HbAlc level of $\geq 6.5 \%$.

Nondiabetic patients with ESRD were included in the CIC-NDRD group $(n=80)$ after screening of patient records and medications along with laboratory testing for plasma glucose or HbA1c levels to exclude diabetes.

Out of the whole cohort, 175 patients were on hemodialysis including 90 diabetic patients and 85 NDRD patients. Patients were dialyzed using a blood flow rate of $200-300 \mathrm{ml} / \mathrm{min}$ and a dialysate flow rate of approximately $500 \mathrm{ml} / \mathrm{min}$.

Estimated GFR was calculated based on the MDRD formula [23]. Serum was used to measure $\mathrm{CN}-1$ activity and concentration. Genotyping was performed on EDTA blood. All samples were stored at $-20^{\circ} \mathrm{C}$ until use. The study protocol was approved by the local ethics committee, and all patients gave written informed consent prior to the study enrollment (no. 0193/2001).

2.2. Genotyping. Genomic DNA was isolated from whole blood using the Genomic DNA isolation kit (Promega, Mannheim, Germany) according to the manufacturer's instruction and stored at $-20^{\circ} \mathrm{C}$ until use. A 167-base pair fragment spanning the $(\mathrm{CTG})_{n}$ polymorphism of CNDP1 was amplified by standard PCR methods using a fluorescence 


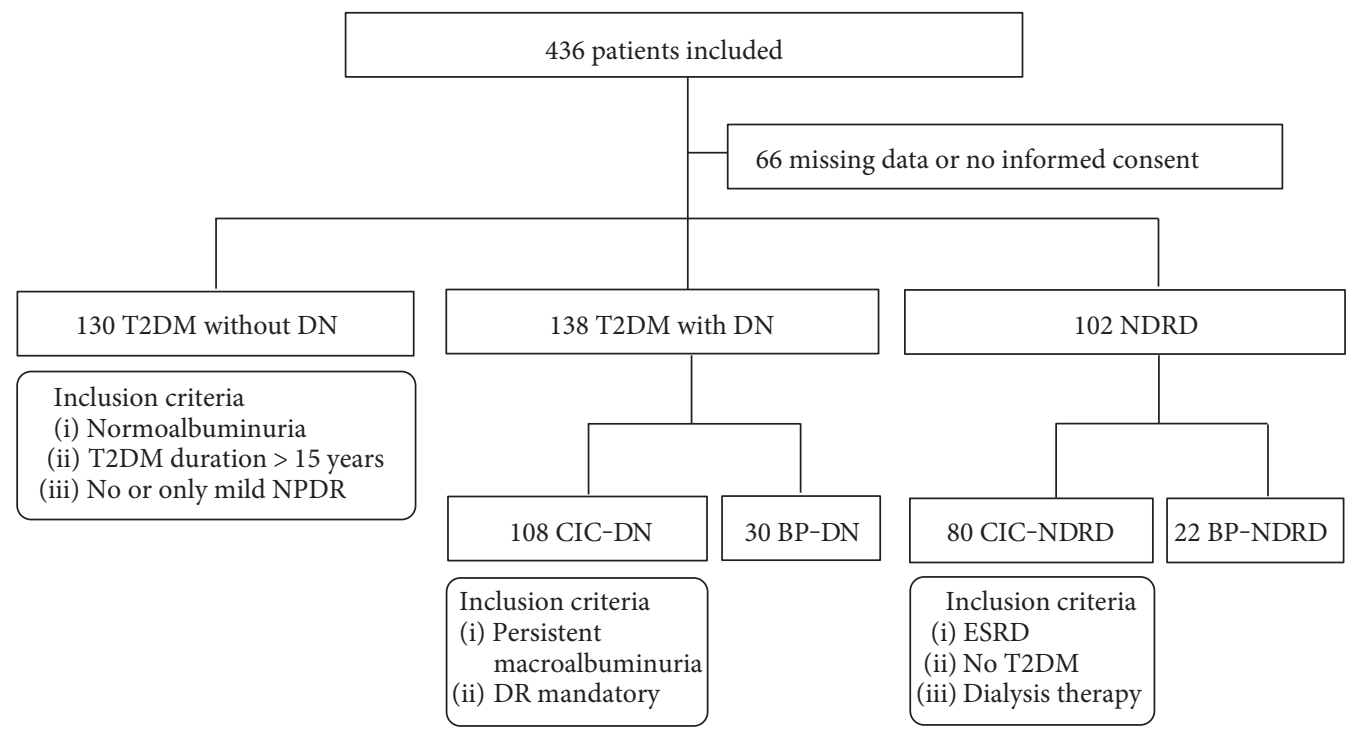

FIGURE 1: Flow diagram for patient recruitment and group allocation. DN: diabetic nephropathy, NDRD: nondiabetic renal disease, NPDR: nonproliferative diabetic retinopathy, CIC: clinical inclusion criteria, BP: biopsy-proven, DR: diabetic retinopathy, ESRD: end-stage renal disease.

labeled forward primer (5'FAM-AGGCAGCTGTGTGAGG TAAC-3') and an unlabeled reverse primer (5'-GGGTGAG GAGAACATGCC-3'), respectively. Genotyping was performed by means of fragment analysis on an ABI 310 sequencing platform (ABI PRISM DNA analyzer 3100).

2.3. CN-1 Activity Assay. CN-1 activity was assayed based on the method described by Teufel et al. [24].

2.4. Statistical Analysis. Quantitative data are depicted as median with corresponding 25th and 75th percentiles (interquartile range) or, when appropriate, as mean \pm SEM. Student's $t$-test was carried out for comparison of continuous variables. Qualitative data were analyzed using the $\chi^{2}$ test. For pairwise comparisons, frequency tables were partitioned into respective $2 \times 2$ subtables. The significance level was corrected using the Bonferroni method based on the number of planned comparisons. Univariate and multivariate logistic regression analyses were performed to examine predictors of biopsy-proven diabetic nephropathy. Variables with a $P$ value of $<0.25$ in the univariate analysis were included into a full-model multivariate analysis. To compare frequencies among groups, which have an ordering, the $\chi^{2}$ test for trend (Cochran-Armitage test for trend) was carried out. Time on hemodialysis was logarithmically transformed before the correlation analysis because of its skewed distribution. The significance level $\alpha$ was defined as 0.05 . Statistical analyses were performed with GraphPad Prism 6.0 (GraphPad Software, Inc., La Jolla, California) and Microsoft Excel/XLSTAT 19.01 (Addinsoft, New York, USA).

\section{Results}

3.1. Patient Characteristics. Demographic and clinical characteristics of all studied individuals are presented in Table 1. Significantly more male patients (64\%) were recruited in the
CIC-DN group (males: $n=69$, females: $n=39$ ) as compared to the no-DN group (50\%) (males: $n=65$, females: $n=65$ ). Gender distribution in the other groups resembled the CICDN group (CIC-NDRD: 61\% males, BP-DN: 70\% males and BP-NDRD: 64\% males). BP-NDRD patients were diagnosed with hypertensive nephrosclerosis, IgA nephropathy, lupus nephritis, or other categories of glomerulonephritis (e.g., granulomatosis with polyangiitis and minimal change GN). Irrespective of subgroup analyses, the distribution of the most prevalent ethnicities was comparable in the no-DN and BP-DN groups as determined by means of $\chi^{2}$ test. Also, the frequency of (CTG) 5 homozygosity did not differ between the two major ethnicities, German and Turkish.

It should be noted that in the group of BP-DN, DM duration was shorter (time from DM diagnosis: 14 (9-20) years versus 21.0 (15-29) years, BP-DN versus CICDN) with more severe hyperglycemia and albuminuria (HbAlc: 7.6 (6.7-8.8) \% versus 7.3 (6.8-8.2) \%, albuminuria: 2070 (337-3290) $\mathrm{mg} / \mathrm{l}$ versus 644 (327-2110) $\mathrm{mg} / \mathrm{l}, \mathrm{BP}-\mathrm{DN}$ versus CIC-DN).

3.2. Association of the CNDP1 (CTG) ${ }_{5}$ Homozygous Genotype with Diabetic Nephropathy. If diagnosis of DN was based on CIC alone, the frequency of the homozygous (CTG) 5 genotype did not significantly differ between the no-DN and CIC-DN groups (Figure 2(a), 36\% versus 38\%). The frequency of the protective genotype dropped to $17 \%$ when biopsy-proven DN was considered only (36\% versus $17 \%$; no-DN versus BP-DN, $P<0.05$, NS after Bonferroni correction) (Figure 2(a)).

To confirm the previous findings of the sex-specific association between $\mathrm{DN}$ and the homozygous $(\mathrm{CTG})_{5}$ genotype, patients were stratified according to gender. In male patients, neither CIC-DN nor BP-DN was associated with (CTG) homozygosity when compared to no-DN (no-DN: $34 \%$, CIC-DN: 42\%, BP-DN: 24\%) (Figure 2(b)). Although the 
TABLE 1: Demographic and clinical data of all patients.

\begin{tabular}{|c|c|c|c|c|c|}
\hline & No-DN & CIC-DN & BP-DN & CIC-NDRD & BP-NDRD \\
\hline$N$ & 130 & 108 & 30 & 80 & 22 \\
\hline \multicolumn{6}{|l|}{ Demographic characteristics } \\
\hline Male sex- $n(\%)$ & $65(50)$ & $69(64)$ & $21(70)$ & $49(61)$ & $14(64)$ \\
\hline Age-year & $71(63-75)$ & $71(62-76)$ & $61(57-69)$ & $61(48-74)$ & $61(55-78)$ \\
\hline \multicolumn{6}{|l|}{ Clinical characteristics } \\
\hline Body mass index $-\mathrm{kg} / \mathrm{m}^{2}$ & $31.1(28-35)$ & $29.8(27-35)$ & $29.9(25-35)$ & $24.5(21-27)$ & $27(24-32)$ \\
\hline \multicolumn{6}{|l|}{ Hypertension } \\
\hline Number of AHM & $3(2-3)$ & $3(2-4)$ & $3(2-5)$ & $3(1.5-4)$ & $2(2-4)$ \\
\hline \multicolumn{6}{|l|}{ Blood pressure $-\mathrm{mmHg}$} \\
\hline Systolic & $129(120-140)$ & $135(120-156)$ & $140(130-150)$ & $135(120-145)$ & $140(128-153)$ \\
\hline Diastolic & $70(66-80)$ & $70(60-80)$ & $75(70-80)$ & $70(60-80)$ & $70(64-80)$ \\
\hline \multicolumn{6}{|l|}{ Diabetes mellitus } \\
\hline Time from diagnosis-year & $16(13-20)$ & $21(15-29)$ & $14(9-20)$ & - & - \\
\hline $\mathrm{HbAlc}-\%$ & $7.0(6.4-8.1)$ & $7.3(6.8-8.2)$ & $7.6(6.7-8.8)$ & $5.6(5.4-5.7)$ & $5.6(5.1-6.2)$ \\
\hline \multicolumn{6}{|l|}{ Kidney function } \\
\hline Creatinine $-\mathrm{mg} / \mathrm{dl}$ & $0.9(0.8-1.1)$ & $6.3(3.7-8.8)$ & $5.7(3.0-7.3)$ & $9.7(7.5-11.5)$ & $3.4(1.7-5.0)$ \\
\hline $\mathrm{eGFR}-\mathrm{ml} / \mathrm{min}$ & $73(61-87)$ & $9(6-16)$ & $10(6-20)$ & $5(4-8)$ & $15(8-37)$ \\
\hline Hemodialysis- $n(\%)$ & $0(0)$ & $83(75)$ & $18(60)$ & $85(100)$ & $8(36)$ \\
\hline HD duration-months* & $0(0-0)$ & $26(1-69)$ & $3(0-30)$ & $56(28-100)$ & $0(0-0.3)$ \\
\hline Albuminuria-mg/l & $9(5-16)$ & $644(327-2110)$ & $2070(337-3290)$ & $470(261-1587)$ & $556(189-1308)$ \\
\hline \multicolumn{6}{|l|}{ Retinopathy $(D R)-n(\%)$} \\
\hline No DR & $107(82)$ & $1(0)$ & $6(20)$ & - & - \\
\hline NPDR & $23(18)$ & $68(63)$ & $11(37)$ & - & - \\
\hline Proliferative DR & - & $17(16)$ & $4(13)$ & - & - \\
\hline Maculopathy & - & $8(7)$ & $3(10)$ & - & - \\
\hline Laser therapy & - & $13(12)$ & $3(10)$ & - & - \\
\hline Polyneuropathy $-n(\%)$ & $54(42)$ & $56(52)$ & $17(57)$ & - & - \\
\hline \multicolumn{6}{|l|}{ History-n $(\%)$} \\
\hline Coronary heart disease & $42(32)$ & $74(69)$ & $11(37)$ & $24(30)$ & $6(22)$ \\
\hline Cardiovascular event & $20(15)$ & $42(39)$ & $7(23)$ & $14(18)$ & $5(23)$ \\
\hline Arterial occlusive disease & $24(18)$ & $56(52)$ & $11(37)$ & $17(21)$ & $3(14)$ \\
\hline Stroke & $19(15)$ & $28(26)$ & $6(20)$ & $8(10)$ & $2(9)$ \\
\hline Statin & $86(66)$ & $79(73)$ & $19(63)$ & $28(35)$ & $10(45)$ \\
\hline Homozygous $\mathrm{CTG}_{5}-n(\%)$ & $47(36)$ & $41(38)$ & $5(17)$ & $32(40)$ & $7(32)$ \\
\hline
\end{tabular}

${ }^{*}$ Patients on hemodialysis only. Categorical data are represented as numbers (\%) and continuous data as median with corresponding 25 th and 75 th percentiles (IQR). AHM: antihypertensive medication; eGFR: estimated glomerular filtration rate; HD: hemodialysis; DR: diabetic retinopathy; NPDR: nonproliferative diabetic retinopathy.

homozygous $(\mathrm{CTG})_{5}$ genotype was less frequent in both the female CIC-DN and the female BP-DN groups as compared to the no-DN group, this difference was only significant for BP-DN (38\% versus $0 \%$, no-DN versus BP-DN, $P<0.05$, significant after Bonferroni correction) (Figure 2(c)).

To confirm that (CTG) $)_{5}$ homozygosity is an independent, negative predictor for biopsy-proven diabetic nephropathy, multivariate logistic regression analysis was performed (Table 2). Seven diabetes-associated factors were selected as independent variables (age, BMI, diabetes duration, HbA1c, male sex, systolic blood pressure, and $(\mathrm{CTG})_{5}$ homozygosity). All variables except BMI showed a $P$ value below 0.25 in univariate analysis and were consequently included in the multivariate model. The HosmerLemeshow test demonstrated an excellent goodness of fit $\left(\chi^{2}=3949, P=0.862\right)$ of the resulting multivariate model. The area under the receiver operating characteristic curve (ROC-AUC) further indicated adequate discrimination $(\mathrm{AUC}=0.797)$.

The $(\mathrm{CTG})_{5}$ homozygous genotype was significantly associated with biopsy-proven nephropathy in both univariate $(\mathrm{OR}=0.353, P=0.047)$ and multivariate analyses $(\mathrm{OR}=0.307, P=0.046)$ and, as such, identified as an independent protective factor. Interestingly, significance was reached despite the markedly lower number of cases $(n=30)$ generally 


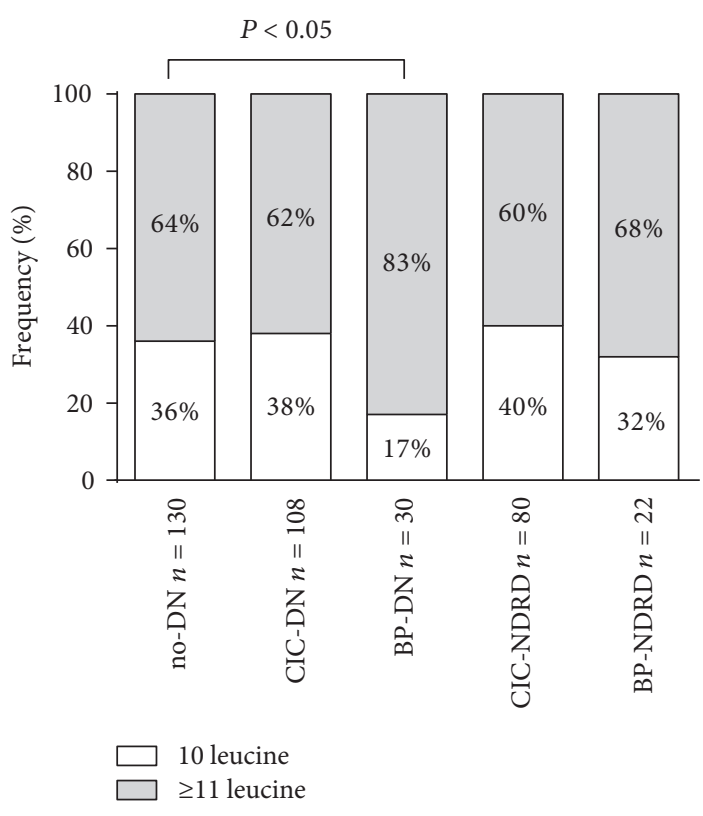

(a)

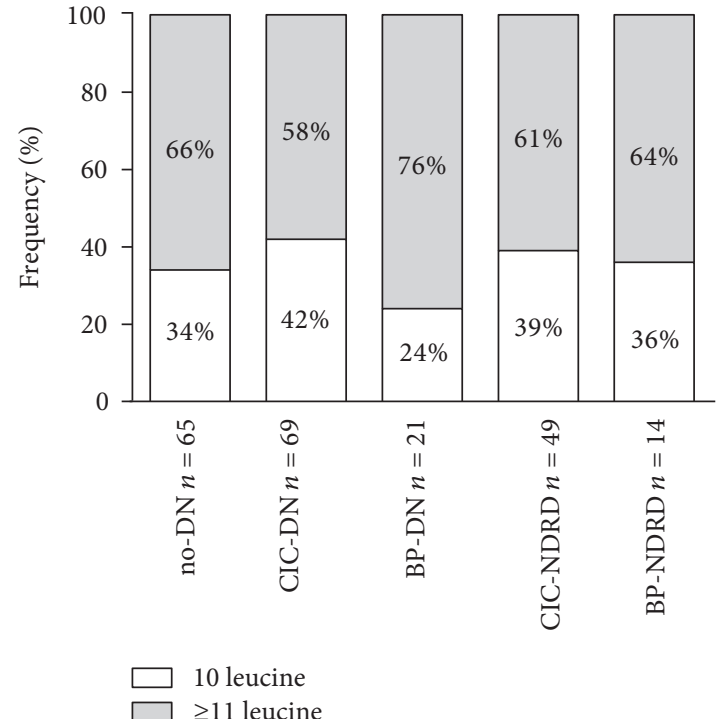

(b)

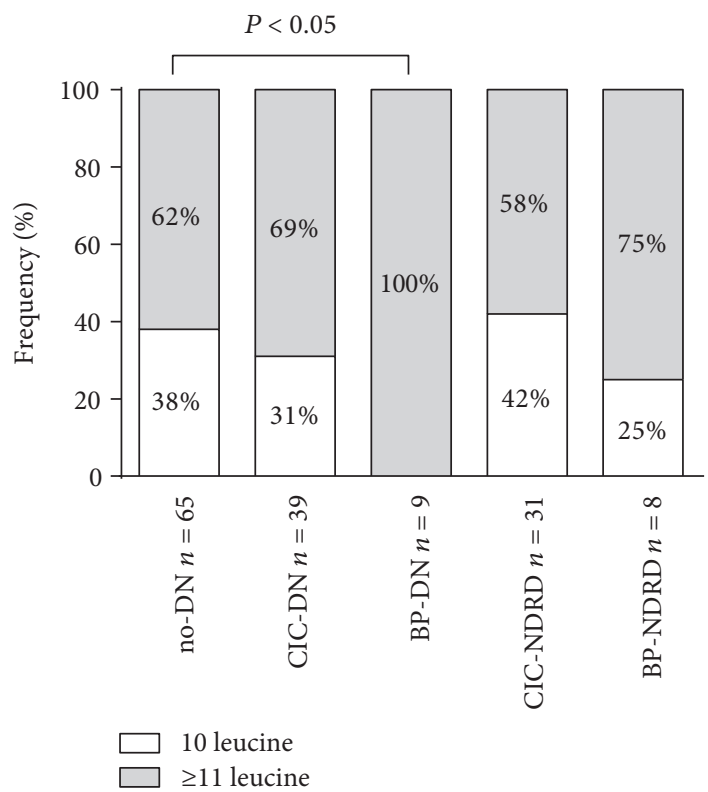

(c)

FIGURE 2: CNDP1 (CTG) ${ }_{n}$ genotype distribution in T2DM patients. Genotype distribution is depicted as homozygosity for the (CTG) $)_{5}$ allele (10 leucine) versus all other genotypes ( $\geq 11$ leucine). Planned comparisons were carried out between T2DM patients without DN and with either CIC- or BP-defined nephropathy. (a) No significant difference in genotype distribution was observed between T2DM patients with DN and without DN when applying CIC. The frequency of patients homozygous for the (CTG) $)_{5}$ allele decreased when BP-DN was considered. However, this difference did not hold after Bonferroni correction. ((b) and (c)) Gender stratification ((b) male patients, (c) female patients) showed no significant difference in the frequency of homozygosity for the CNDP1 (CTG) $)_{5}$ allele between T2DM with and without DN when applying CIC. When DN was confirmed through biopsy, however, the frequency of CNDP1 (CTG) ${ }_{5}$ homozygosity significantly decreased in female T2DM patients, which remained significant after Bonferroni adjustment.

believed to be necessary for obtaining sufficient power (i.e., $n=10$ /independent variable). In the multivariate model, systolic blood pressure $(\mathrm{OR}=1.024, P=0.045)$ was positively and age $(\mathrm{OR}=0.931, P=0.007)$ and diabetes duration $(\mathrm{OR}=0.895, P=0.025)$ were negatively associated with biopsy-proven nephropathy, respectively.
3.3. CNDP1 Genotype Distribution over Time on Hemodialysis and Diabetes Duration. The CNDP1 genotype distribution $\left(C N D P 1 \quad(\mathrm{CTG})_{5}\right.$ homozygous-versus all other CNDP1 genotypes) was tested in 175 patients on hemodialysis, including 90 patients with DN according to CIC and/or BP-DN and 85 CIC-NDRD patients. Patients were stratified 
TABLE 2: Summary of logistic regression analysis of variables predicting biopsy-proven diabetic nephropathy (male and female, $n=160$ ).

\begin{tabular}{|c|c|c|c|c|c|c|}
\hline & \multicolumn{3}{|c|}{ Univariate analysis } & \multicolumn{3}{|c|}{ Multivariate analysis } \\
\hline & OR & $95 \%$ CI & $P$ value & OR & $95 \%$ CI & $P$ value \\
\hline Age (years) & 0.920 & $0.881-0.961$ & $<0.001$ & 0.931 & $0.883-0.980$ & 0.007 \\
\hline BMI $\left(\mathrm{kg} / \mathrm{m}^{2}\right)$ & 0.980 & $0.922-1.041$ & 0.513 & - & - & - \\
\hline Diabetes duration (years) & 0.886 & $0.813-0.966$ & 0.006 & 0.895 & $0.812-0.986$ & 0.025 \\
\hline HbAlc (\%) & 1.308 & $1.020-1.678$ & 0.034 & 1.169 & $0.844-1.619$ & 0.348 \\
\hline Male sex & 2.333 & $0.994-5.477$ & 0.052 & 1.957 & $0.748-5.124$ & 0.171 \\
\hline SBP (mmHg) & 1.021 & $1.000-1.042$ & 0.052 & 1.024 & $1.001-1.047$ & 0.045 \\
\hline $\mathrm{CTG}_{5}$ homozygosity & 0.353 & $0.127-0.984$ & 0.047 & 0.307 & $0.096-0.980$ & 0.046 \\
\hline
\end{tabular}

Area under the ROC curve (AUC) $=0.797, P=0.862$ for Hosmer-Lemeshow test. BMI: body mass index; SBP: systolic blood pressure.

on the basis of hemodialysis duration, that is, time on dialysis: $<30$ months $(n=60)$, between 30 and 100 months $(n=76)$ and $>100$ months $(n=39)$. To assess the frequencies over time on hemodialysis and diabetes duration, the $\chi^{2}$ test for trend was carried out.

The frequency of the $(\mathrm{CTG})_{5}$ homozygous genotype significantly increased with time on hemodialysis (<30 months: 33\%, 30-100 months: 40\%, >100 months: 49\%, $P<0.05$ ) (Figure 3(a)), while gender distribution was approximately equal in the groups. The association of the (CTG) homozygous genotype with time on hemodialysis was not significant in the subgroup analyses after gender stratification (contingency tables not shown).

Although the frequency of the homozygous CNDP1 $(\mathrm{CTG})_{5}$ genotype uniformly increased with diabetes duration, this trend did not reach statistical significance $(<10$ years: $27 \%, 10-15$ years: $34 \%, 16-20$ years: $37 \%$, $>20$ years: $42 \%, P=0.17$ ) (Figure 3(b)). However, if patients with a heterozygous CNDP1 (CTG) $)_{5}$ genotype were included (i.e., patients with at least one $(\mathrm{CTG})_{5}$ allele), there was a clear, significant trend towards high frequencies with increasing diabetes duration ( $<10$ years: $64 \%, 10-15$ years: $75 \%, \quad 16-20$ years: $87 \%, \quad>20$ years: $90 \%, \quad P<0.01)$ (Figure 3(c)).

3.4. CN-1 Activities in Hemodialysis Patients. Because serum carnosinase $(\mathrm{CN}-1)$ activity correlates with $C N D P 1(\mathrm{CTG})_{n}$ genotypes, that is, $\mathrm{CN}-1$ activity is in general lower in individuals with less CTG copies, we also cross-sectionally assessed if serum $\mathrm{CN}-1$ activity changes with time on dialysis. In line with the increased frequency of the homozygous CNDP1 (CTG) 5 genotype in the groups of patients with a long history of hemodialysis, a significant negative correlation between serum $\mathrm{CN}-1$ activity and log-transformed hemodialysis duration was found in all patients $(r=-0.33$; $P<0.0001$, Figure 4(a)), T2DM patients only $(r=-0.034$, $P=0.0006$, Figure 4(b)), and nondiabetic hemodialysis patients $(r=-0,031, P=0.004$, Figure $4(c))$.

To delineate if the CNDP1 genotype is relevant for CN-1 activities in hemodialysis patients, patients on hemodialysis were stratified on the basis of homozygosity for the $(\mathrm{CTG})_{5}$ allele. Out of 174 subjects, the 65 patients carrying the homozygous CNDP1 (CTG) $)_{5}$ genotype showed a significantly lower serum $\mathrm{CN}-1$ activity compared to patients with other genotypes (Figure 5(a), $P<0.01$ ). This remained significant in female (Figure 5(c), $P<0.05$ ), but not in male (Figure 5(b), $P=0.07$ ) patients after gender stratification.

\section{Discussion}

This study examined whether the protection against DN afforded by the homozygous $C N D P 1$ (CTG) ${ }_{5}$ genotype is still observed when applying clinical inclusion criteria or biopsy findings only and if the prevalence of the protective genotype changes in situations of increased cardiovascular mortality. Our results demonstrate that the frequency of the homozygous CNDP1 (CTG) $)_{5}$ genotype in the group of patients with biopsy-proven $\mathrm{DN}$ is significantly lower as compared to the groups of patients with no DN or with other biopsy-proven nephropathies. Our study also indicates that the frequency of homozygous CNDP1 (CTG) 5 genotype tends to be higher in patients with a longer duration of hemodialysis, particularly in female patients. An analogous increase was detected for patients carrying at least one $(\mathrm{CTG})_{5}$ allele stratified for diabetes duration.

CIC for group allocations in DN studies bears the risk of wrongly assigning patients to the DN group as up to $20-50 \%$ of diabetic patients with albuminuria develop NDRD without concurrent DN $[25,26]$. Although the presence of diabetic retinopathy (DR) is helpful for the prediction of DN [27] and thus improves the validity of group allocation, still DR may be absent in up to $50 \%$ of DN patients $[28,29]$. Controversial studies reporting on genetic susceptibility loci for DN, including those for the CNDP1 (CTG) ${ }_{n}$ polymorphism, might partly underlie this problem.

The use of large cohorts from different consortia and subsequent meta-analysis of data obtained from genome-wide association studies (GWAS) may partly overcome this problem as the proportion of wrongly allocated patients might be outnumbered by the large number of studied patients. The GWAS approach has been successfully utilized in newer studies confirming susceptibility loci for declining glomerular filtration rate (eGFR) or albuminuria [30-32]. The CNDP1 locus, a postulated DN susceptibility locus found by positional cloning [5] and case control studies [10], has 


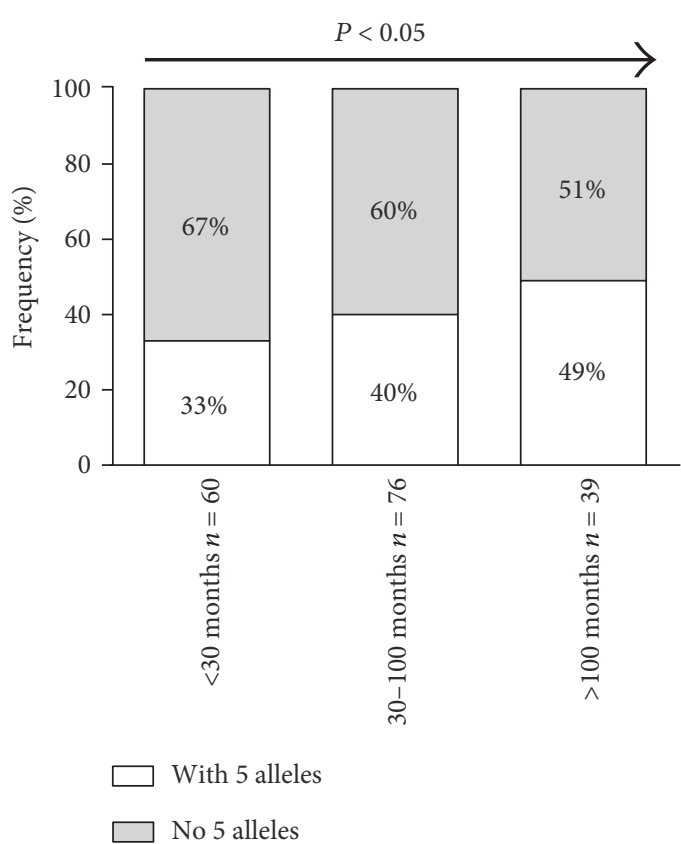

(a)

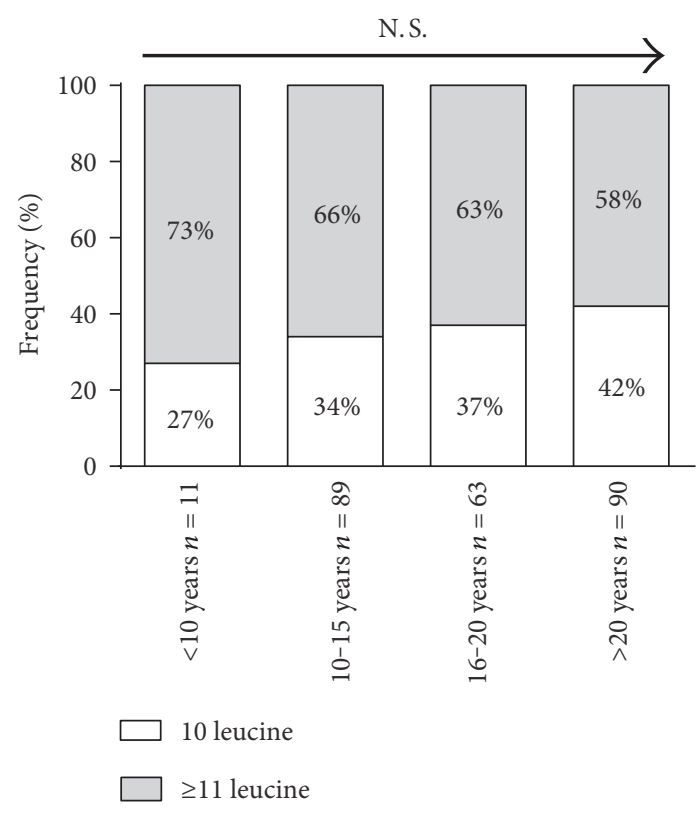

(b)

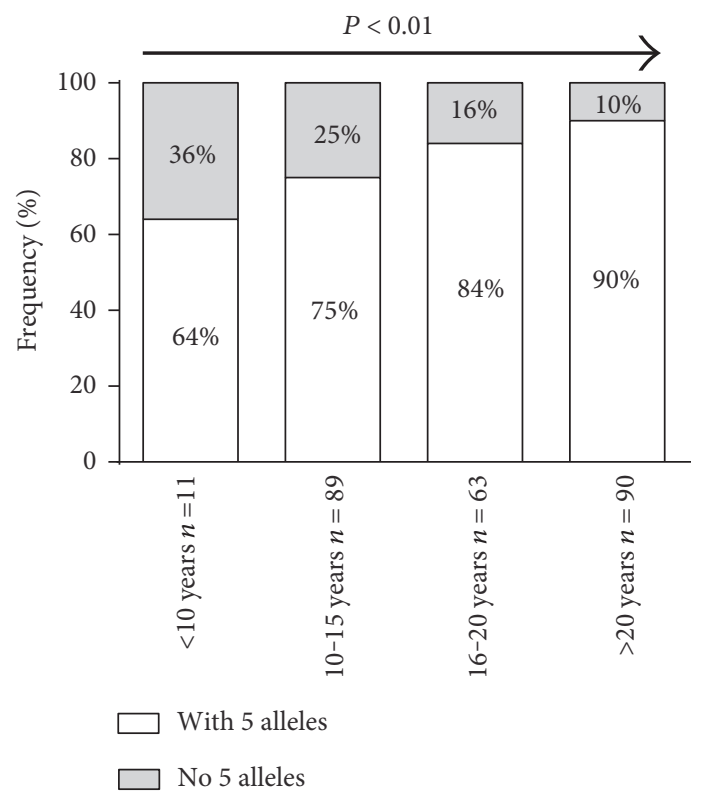

(c)

Figure 3: The CNDP1 (CTG) ${ }_{5}$ genotype distribution is changed with time on dialysis and diabetes duration. To assess if the frequencies change over time, the $\chi^{2}$ test for trend (Cochran-Armitage test for trend) was carried out. (a) The frequency of the homozygous CNDP1 $(\mathrm{CTG})_{5}$ genotype (10 leucine) significantly increased with time on hemodialysis. ((b) and (c)) Although the observed frequency of the homozygous (b) CNDP1 (CTG) 5 genotype uniformly increased with diabetes duration, this trend did not reach statistical significance. Yet, if patients with a heterozygous (c) CNDP1 (CTG) $)_{5}$ genotype (one 5 allele) were included as well, a significant trend towards high frequencies with increasing diabetes duration was found. N.S.: not significant.

never been reported to be linked to DN in a GWAS approach, despite the fact that other genetic studies $[11,14,33,34]$ including a meta-analysis on 4546 DN, 7994 diabetes mellitus (DM), and 1826 healthy subjects [35] have confirmed an association between the CNDP1 $(\mathrm{CTG})_{n}$ polymorphism and $\mathrm{DN}$ in T2DM patients. Significance further increased in these studies if more stringent CIC for DN, for example, the presence of proliferative DR and a longer duration of T2DM, were considered [11].

In our study, the association between the protective homozygous $C N D P 1(\mathrm{CTG})_{5}$ genotype and $\mathrm{DN}$ was restricted to biopsy-proven $\mathrm{DN}$ and only significant in female patients using the $\chi^{2}$ test. Multivariate logistic regression subsequently identified $(\mathrm{CTG})_{5}$ homozygosity as an independent protective 


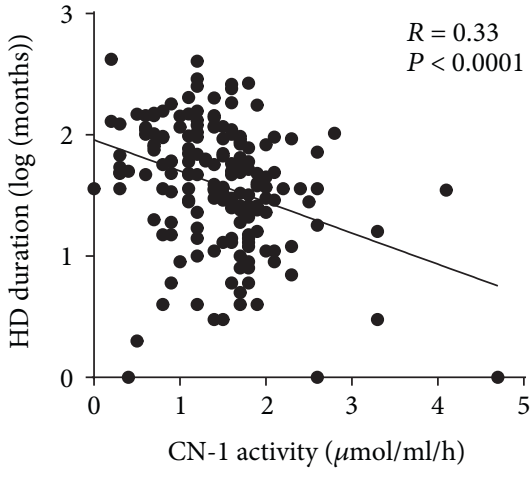

(a)

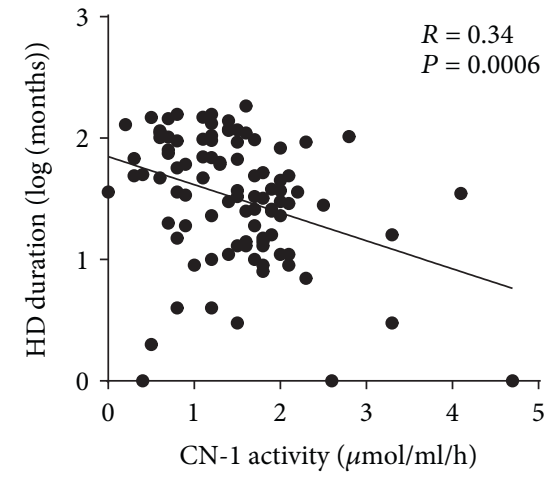

(b)

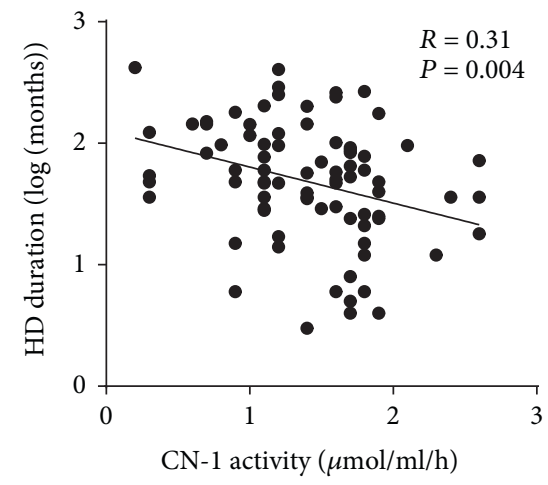

(c)

FIgURE 4: CN-1 activities decrease with time on dialysis. (a) Serum CN-1 activity was assessed in 175 hemodialysis patients and plotted against the log-transformed duration since hemodialysis was initiated. A significant correlation between serum CN-1 activity and logtransformed hemodialysis duration was found in all patients. ((b) and (c)) After stratification in T2DM (b) and other causes of renal failure (c), the correlation remained significant. HD: hemodialysis.

factor for biopsy-proven $\mathrm{DN}$ with an odds ratio of approximately 0.3 . The negative association of age and diabetes duration with biopsy-proven $\mathrm{DN}$ in this analysis may be explained by the fact that in older diabetic patients, a renal biopsy is often waived due to the lack of consequence.

The frequencies of the homozygous CNDP1 (CTG) genotype in the no-DN and BP-NDRD groups were comparable, suggesting that this genotype does not afford protection against NDRD. Nonetheless, it would be prudent to be cautious with this assumption as other studies have suggested that this genotype also affords protection against other chronic kidney diseases (CKD), for example, glomerulonephritis but not tubulointerstitial nephritis [36]. In this light, the paradox between CIC-DN and BP-DN might be due to the fact that patients with NDRD were falsely included in the CIC-DN group, underscoring that clinical criteria do not provide a sufficient certainty for the diagnosis of $\mathrm{DN}$ in T2DM patients. It is important to note that patients in the BP-DN group had a shorter DM duration and displayed more severe hyperglycemia and albuminuria as compared to the CIC-DN group. Because of this relatively atypical DN course, these patients required a biopsy to clarify the actual underlying renal disease. Whether the change in CNDP1 genotype distribution between CIC-DN and BP-DN underlies the severity of disease per se is unknown so far and cannot be excluded.
Our data are in agreement with a previous publication showing that the association of CNDP1 and DN is most likely sex-specific [14]. Although also in males of the BP-DN group the frequency of the $(\mathrm{CTG})_{5}$ homozygous genotype was lower as compared to the no-DN group, it was only significantly decreased in female BP-DN patients. The sex-specific protection of CNDP1 is generally explained by higher serum $\mathrm{CN}-1$ activities in females [14].

In keeping with the recently published prospective study that the CNDP1 genotype may impart a cardiovascular mortality risk in female, but not in male T2DM patients [19], we investigated whether genotype distribution changes with time on dialysis or diabetes duration. Since both of the latter variables are associated with an increased (cardiovascular) mortality risk, it would be expected based on the above study that the frequency of the homozygous CNDP1 (CTG) $)_{5}$ genotype would decrease rather than increase in patients with a long history of hemodialysis. By contrast, our data show that the frequency of the homozygous CNDP1 (CTG) ${ }_{5}$ genotype was significantly increased in patients with a long history of hemodialysis. This difference remained in both males and females although it was not statistically significant, which is likely explained by the small sample size of the subgroup analysis. Similar findings were also observed with respect to a longstanding diabetes duration when all patients carrying at least one $(\mathrm{CTG})_{5}$ allele were included. 


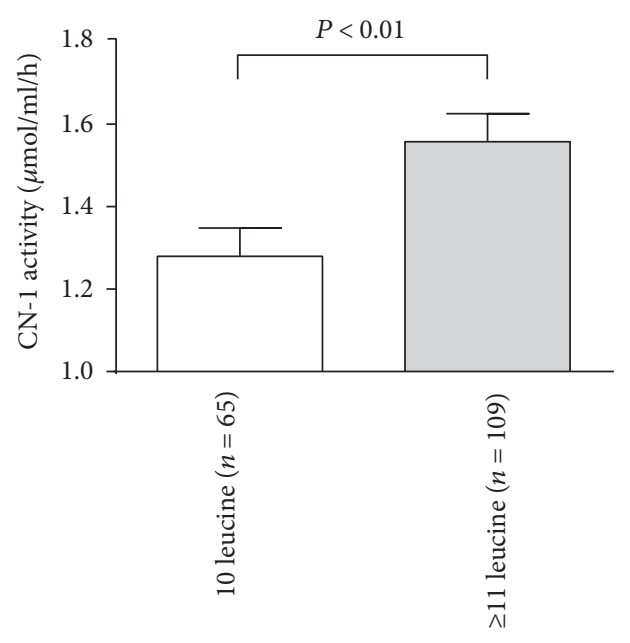

(a)

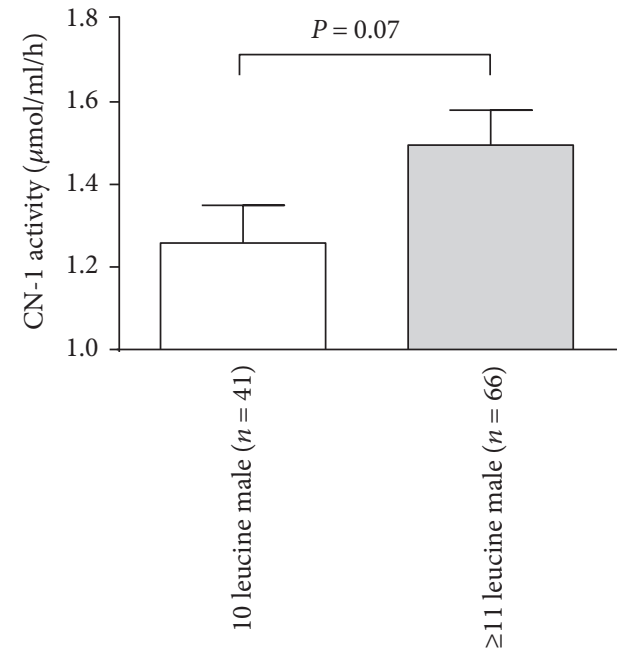

(b)

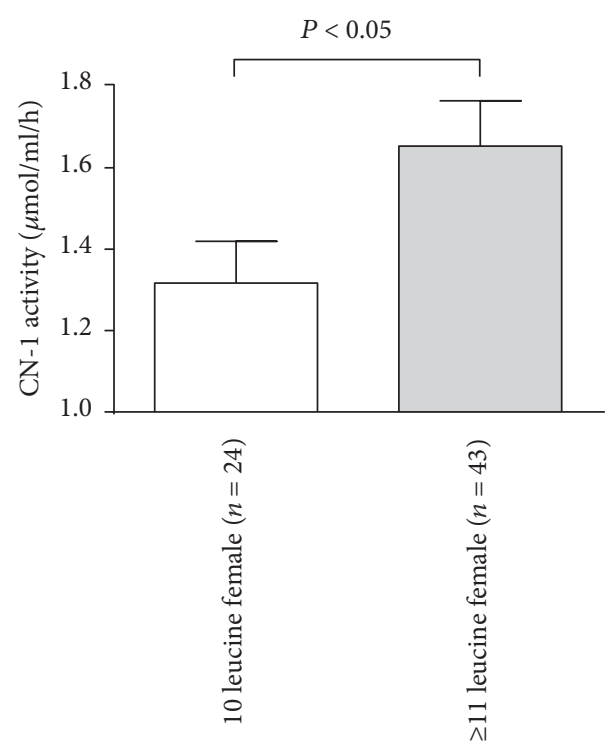

(c)

FIgURE 5: CN-1 activity correlates with CNDP1 genotype in hemodialysis patients. (a) CN-1 activity in (CTG) 5 homozygous hemodialysis patients is significantly lower than that in patients carrying other genotypes. ((b) and (c)) After gender stratification differences in $\mathrm{CN}-1$ activity between the $(\mathrm{CTG})_{5}$ homozygous, all other genotypes remained but only reached statistical significance in females (c).

Although our findings suggest that in patients on hemodialysis and in diabetic patients the CNDP1 (CTG) ${ }_{5}$ genotype may not impart an additional mortality risk, it should be underscored that the small group sizes and the cross-sectional design of this study impede drawing firm conclusions, in particular since a considerable number of hemodialysis patients were not diabetic. Nonetheless, these findings suggest that in patients on hemodialysis and in diabetes, the CNDP1 (CTG) 5 genotype may not impart an additional mortality risk.

Serum CN-1 concentrations and activities are in part determined by $(\mathrm{CTG})_{n}$ polymorphism $[10,37]$. Since this repeat is located in the hydrophobic part of the $\mathrm{CN}-1$ signal peptide and is essential for the translocation of $\mathrm{CN}-1$ protein during secretion, it is believed that the shorter (CTG) variant is less efficiently secreted [37]. In line with an increased frequency of the homozygous CNDP1 (CTG) genotype in patients with a long history of hemodialysis, $\mathrm{CN}-1$ activities were reduced. This reduction is not due to the loss of protein through hemofiltration since serum $\mathrm{CN}-1$ concentrations even increase proportionally to the amount of ultrafiltrate [38].

As discussed above, we acknowledge the relatively small sample size as a major limitation of our study, accounting for a limited statistical power regarding major questions addressed. In addition, in contrast to a prospective study design, no systematic biopsy strategy with uniform indications and assigned nephropathologists could be implemented. Other studies, however, demonstrated that the histological classification of $\mathrm{DN}$ based on glomerulopathy shows a satisfying interobserver reproducibility [39]. We also acknowledge uncertainties regarding the 
procedure of patient allocation leading to limited group selectivity. This holds true especially for the diabetic control group without DN, which is based only on clinical criteria instead of a histological diagnosis. As $80 \%$ of these patients were on $\mathrm{ACE}$ inhibitor or $\mathrm{AT}_{1}$-blocking drugs, albuminuria alone was not a reliable parameter. Despite the extension of the criteria by diabetes duration of $>15$ years and exclusion of patients which manifest retinopathy, accidental assignment of patients with $\mathrm{DN}$ to this group is not improbable.

In our eyes, the fact that this study still resulted in significant results in the light of these conceptual drawbacks supports a particular strong association of our findings. Our study supports the hypothesis that protection against $\mathrm{DN}$ is indeed afforded by the CNDP1 (CTG) $)_{5}$ genotype and that this association mainly applies to female T2DM patients. The restriction of this finding to the BP-DN group may be attributed to false allocation of patients with other proteinuric diseases to the CIC-DN group. In fact, $20-50 \%$ of diabetic patients with proteinuria display NDRD without concurrent DN $[25,26]$. Our investigation also suggests that $(\mathrm{CTG})_{5}$ homozygous hemodialysis patients and patients with diabetes carrying at least one $(\mathrm{CTG})_{5}$ allele might have a survival benefit as compared to other genotypes. These findings warrant further conformational studies, ideally with a prospective longitudinal design.

\section{Conflicts of Interest}

The authors declare that they have no conflicts of interest.

\section{Authors' Contributions}

Thomas Albrecht and Shiqi Zhang are equally contributing authors.

\section{Acknowledgments}

This work was supported by the Deutsche Forschungsgemeinschaft (DFG) SA 2143/1-1 to Sibylle J. Hauske and Graduiertenkolleg (GRK) 1874/1 to Benito A. Yard and Sibylle J. Hauske. The authors would like to thank all involved Dialysis Units and Diabetic Centers for supporting the recruitment of patients. The authors would also like to thank Annette Breedijk (University Medical Center Mannheim) for her invaluable technical assistance and David Papke (Harvard Medical School, Boston, USA) for proofreading this manuscript.

\section{References}

[1] C. H. Hasslacher, E. Ritz, P. Wahl, and C. Michael, "Similar risks of nephropathy in patients with type I or type II diabetes mellitus," Nephrology, Dialysis, Transplantation, vol. 4, no. 10, pp. 859-863, 1989.

[2] J. William, D. Hogan, and D. Batlle, "Predicting the development of diabetic nephropathy and its progression," Advances in Chronic Kidney Disease, vol. 12, no. 2, pp. 202-211, 2005.

[3] E. R. Seaquist, F. C. Goetz, S. Rich, and J. Barbosa, "Familial clustering of diabetic kidney disease. Evidence for genetic susceptibility to diabetic nephropathy," The New England Journal of Medicine, vol. 320, no. 18, pp. 1161-1165, 1989.

[4] B. I. Freedman, D. W. Bowden, M. M. Sale, C. D. Langefeld, and S. S. Rich, "Genetic susceptibility contributes to renal and cardiovascular complications of type 2 diabetes mellitus," Hypertension, vol. 48, no. 1, pp. 8-13, 2006.

[5] I. Vardarli, L. J. Baier, R. L. Hanson et al., "Gene for susceptibility to diabetic nephropathy in type 2 diabetes maps to 18q22.3-23," Kidney International, vol. 62, no. 6, pp. 21762183, 2002.

[6] D. W. Bowden, C. J. Colicigno, C. D. Langefeld et al., "A genome scan for diabetic nephropathy in African Americans," Kidney International, vol. 66, no. 4, pp. 1517-1526, 2004.

[7] S. K. Iyengar, H. E. Abboud, K. A. Goddard et al., "Genomewide scans for diabetic nephropathy and albuminuria in multiethnic populations: the family investigation of nephropathy and diabetes (FIND)," Diabetes, vol. 56, no. 6, pp. 15771585, 2007.

[8] H. A. Chakkera, R. L. Hanson, S. Kobes et al., "Association of variants in the carnosine peptidase 1 gene (CNDP1) with diabetic nephropathy in American Indians," Molecular Genetics and Metabolism, vol. 103, no. 2, pp. 185-190, 2011.

[9] C. W. McDonough, P. J. Hicks, L. Lu, C. D. Langefeld, B. I. Freedman, and D. W. Bowden, "The influence of carnosinase gene polymorphisms on diabetic nephropathy risk in African-Americans," Human Genetics, vol. 126, no. 2, pp. 265-275, 2009.

[10] B. Janssen, D. Hohenadel, P. Brinkkoetter et al., "Carnosine as a protective factor in diabetic nephropathy: association with a leucine repeat of the carnosinase gene CNDP1," Diabetes, vol. 54, no. 8, pp. 2320-2327, 2005.

[11] B. I. Freedman, P. J. Hicks, M. M. Sale et al., "A leucine repeat in the carnosinase gene CNDP1 is associated with diabetic end-stage renal disease in European Americans," Nephrology, Dialysis, Transplantation, vol. 22, no. 4, pp. 1131-1135, 2007.

[12] P. Y. Poon, C. C. Szeto, B. C. Kwan, K. M. Chow, and P. K. $\mathrm{Li}$, "Relationship between carnosinase gene CNDP1 leucine repeat polymorphism and the clinical outcome of Chinese PD patients," Clinical Nephrology, vol. 74, no. 5, pp. 343$345,2010$.

[13] A. L. Mooyaart, I. G. van Valkengoed, P. C. Shaw et al., "Lower frequency of the 5/5 homozygous CNDP1 genotype in South Asian Surinamese," Diabetes Research and Clinical Practice, vol. 85, no. 3, pp. 272-278, 2009.

[14] A. L. Mooyaart, A. Zutinic, S. J. Bakker et al., "Association between CNDP1 genotype and diabetic nephropathy is sex specific," Diabetes, vol. 59, no. 6, pp. 1555-1559, 2010.

[15] T. T. Pham, J. J. Sim, D. A. Kujubu, I. L. Liu, and V. A. Kumar, "Prevalence of nondiabetic renal disease in diabetic patients," American Journal of Nephrology, vol. 27, no. 3, pp. 322-328, 2007.

[16] S. Olsen and C. E. Mogensen, "How often is NIDDM complicated with non-diabetic renal disease? An analysis of renal biopsies and the literature," Diabetologia, vol. 39, no. 12 , pp. $1638-1645,1996$.

[17] R. Klein, B. E. Klein, and S. E. Moss, "Epidemiology of proliferative diabetic retinopathy," Diabetes Care, vol. 15, no. 12, pp. 1875-1891, 1992.

[18] A. Chandy, B. Pawar, M. John, and R. Isaac, “Association between diabetic nephropathy and other diabetic 
microvascular and macrovascular complications," Saudi Journal of Kidney Diseases and Transplantation, vol. 19, no. 6, pp. 924-928, 2008.

[19] A. Alkhalaf, G. W. Landman, K. J. van Hateren et al., "Sex specific association between carnosinase gene CNDP1 and cardiovascular mortality in patients with type 2 diabetes (ZODIAC-22)," Journal of Nephrology, vol. 28, no. 2, pp. 201-207, 2015.

[20] H. H. Parving, M. A. Gall, P. Skøtt et al., "Prevalence and causes of albuminuria in non-insulin-dependent diabetic patients," Kidney International, vol. 41, no. 4, pp. 758762, 1992.

[21] M. Kanauchi, T. Kawano, H. Uyama, H. Shiiki, and K. Dohi, "Discordance between retinopathy and nephropathy in type 2 diabetes," Nephron, vol. 80, no. 2, pp. 171-174, 1998.

[22] R. A. Pedro, S. A. Ramon, B. B. Marc, F. B. Juan, and M. M. Isabel, "Prevalence and relationship between diabetic retinopathy and nephropathy, and its risk factors in the north-east of Spain, a population-based study," Ophthalmic Epidemiology, vol. 17 , no. 4, pp. $251-265,2010$.

[23] A. S. Levey, J. P. Bosch, J. B. Lewis, T. Greene, N. Rogers, and D. Roth, "A more accurate method to estimate glomerular filtration rate from serum creatinine: a new prediction equation. Modification of Diet in Renal Disease Study Group," Annals of Internal Medicine, vol. 130, no. 6, pp. 461-470, 1999.

[24] M. Teufel, V. Saudek, J. P. Ledig et al., "Sequence identification and characterization of human carnosinase and a closely related non-specific dipeptidase," The Journal of Biological Chemistry, vol. 278, no. 8, pp. 6521-6531, 2003.

[25] T. Soleymanian, G. Hamid, M. Arefi et al., "Non-diabetic renal disease with or without diabetic nephropathy in type 2 diabetes: clinical predictors and outcome," Renal Failure, vol. 37, no. 4, pp. 572-575, 2015.

[26] S. S. Soni, S. Gowrishankar, A. G. Kishan, and A. Raman, "Non diabetic renal disease in type 2 diabetes mellitus," Nephrology (Carlton, Vic.), vol. 11, no. 6, pp. 533-537, 2006.

[27] F. He, X. Xia, X. F. Wu, X. Q. Yu, and F. X. Huang, "Diabetic retinopathy in predicting diabetic nephropathy in patients with type 2 diabetes and renal disease: a meta-analysis," Diabetologia, vol. 56, no. 3, pp. 457-466, 2013.

[28] G. Wolf, N. Müller, A. Mandecka, and U. A. Müller, “Association of diabetic retinopathy and renal function in patients with types 1 and 2 diabetes mellitus," Clinical Nephrology, vol. 68, no. 2, pp. 81-86, 2007.

[29] J. Prakash, M. Lodha, S. K. Singh, R. Vohra, and R. Raja, "Diabetic retinopathy is a poor predictor of type of nephropathy in proteinuric type 2 diabetic patients," The Journal of the Association of Physicians of India, vol. 55, no. 55, pp. 412-416, 2007.

[30] A. Teumer, A. Tin, R. Sorice et al., "Genome-wide association studies identify genetic loci associated with albuminuria in diabetes," Diabetes, vol. 65, no. 3, pp. 803-817, 2016.

[31] C. A. Böger, M. H. Chen, A. Tin et al., "CUBN is a gene locus for albuminuria," Journal of the American Society of Nephrology, vol. 22, no. 3, pp. 555-570, 2011.

[32] C. Pattaro, A. Teumer, M. Gorski et al., "Genetic associations at 53 loci highlight cell types and biological pathways relevant for kidney function," Nature Communications, vol. 7, Article ID 10023, 2016.
[33] A. Alkhalaf, S. J. Bakker, H. J. Bilo et al., "A polymorphism in the gene encoding carnosinase (CNDP1) as a predictor of mortality and progression from nephropathy to end-stage renal disease in type 1 diabetes mellitus," Diabetologia, vol. 53, no. 12, pp. 2562-2568, 2010.

[34] T. S. Ahluwalia, E. Lindholm, and L. C. Groop, "Common variants in CNDP1 and CNDP2, and risk of nephropathy in type 2 diabetes," Diabetologia, vol. 54, no. 9, pp. 2295-2302, 2011.

[35] J. M. Zhu, B. Wang, J. Li et al., "D18S880 microsatellite polymorphism of carnosinase gene and diabetic nephropathy: a meta-analysis," Genetic Testing and Molecular Biomarkers, vol. 17, no. 4, pp. 289-294, 2013.

[36] K. Kiliś-Pstrusińska, D. Zwolińska, W. Grzeszczak, and S. Group, "Is carnosinase 1 gene (CNDP1) polymorphism associated with chronic kidney disease progression in children and young adults? Results of a family-based study," Archives of Medical Research, vol. 41, no. 5, pp. 356-362, 2010.

[37] E. Riedl, H. Koeppel, P. Brinkkoetter et al., "A CTG polymorphism in the CNDP1 gene determines the secretion of serum carnosinase in Cos-7 transfected cells," Diabetes, vol. 56, no. 9, pp. 2410-2413, 2007.

[38] S. Zhang, H. A. Lindner, S. Kabtni et al., "Monoclonal antibody RYSK173 recognizes the dinuclear $\mathrm{Zn}$ center of serum carnosinase $1(\mathrm{CN}-1)$ : possible consequences of $\mathrm{Zn}$ binding for CN-1 recognition by RYSK173," PLoS One, vol. 11, no. 1, article e0146831, 2016.

[39] T. W. Tervaert, A. L. Mooyaart, K. Amann et al., "Pathologic classification of diabetic nephropathy," Journal of the American Society of Nephrology, vol. 21, no. 4, pp. 556-563, 2010. 


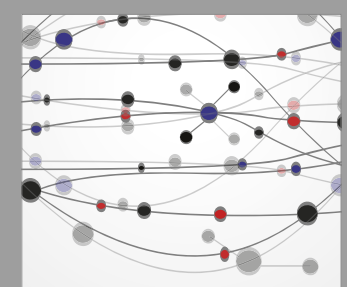

The Scientific World Journal
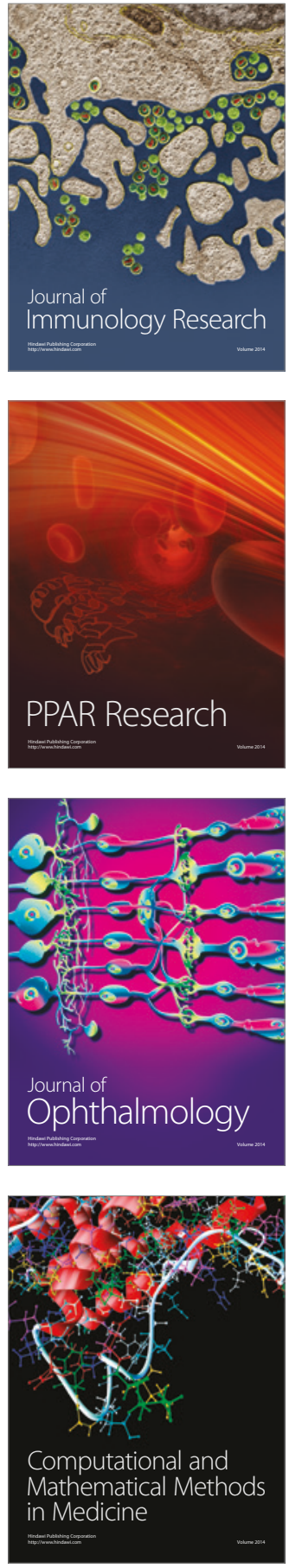

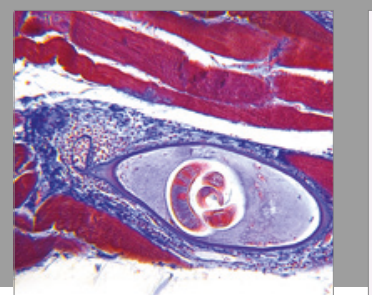

Gastroenterology Research and Practice
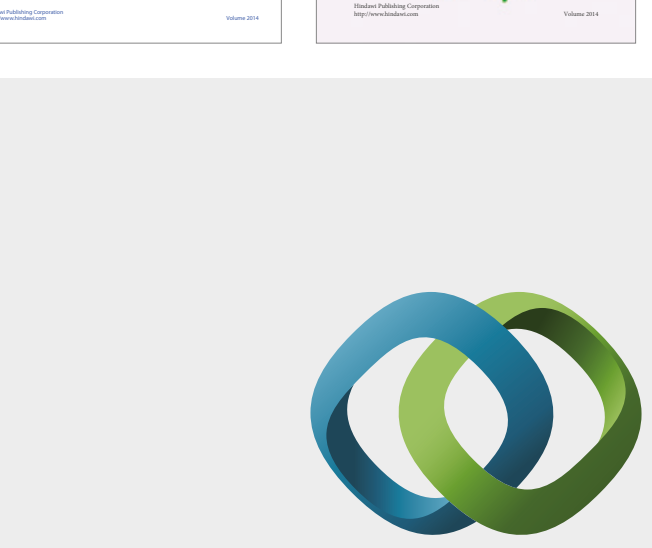

\section{Hindawi}

Submit your manuscripts at

https://www.hindawi.com
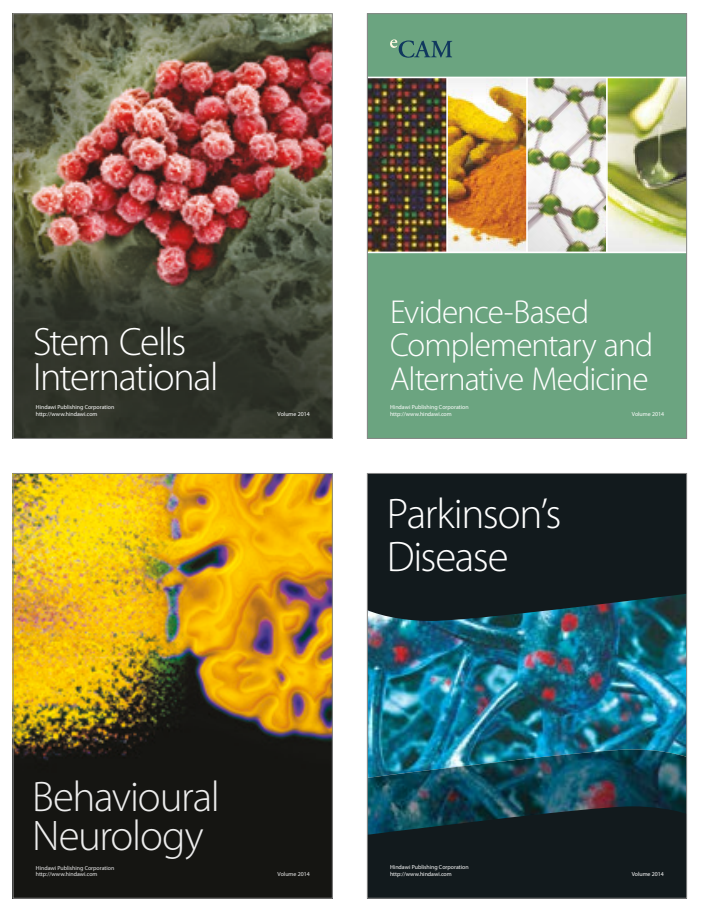
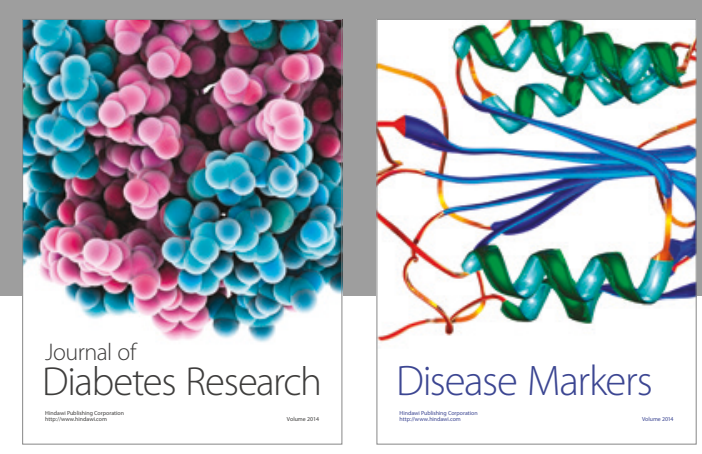

Disease Markers
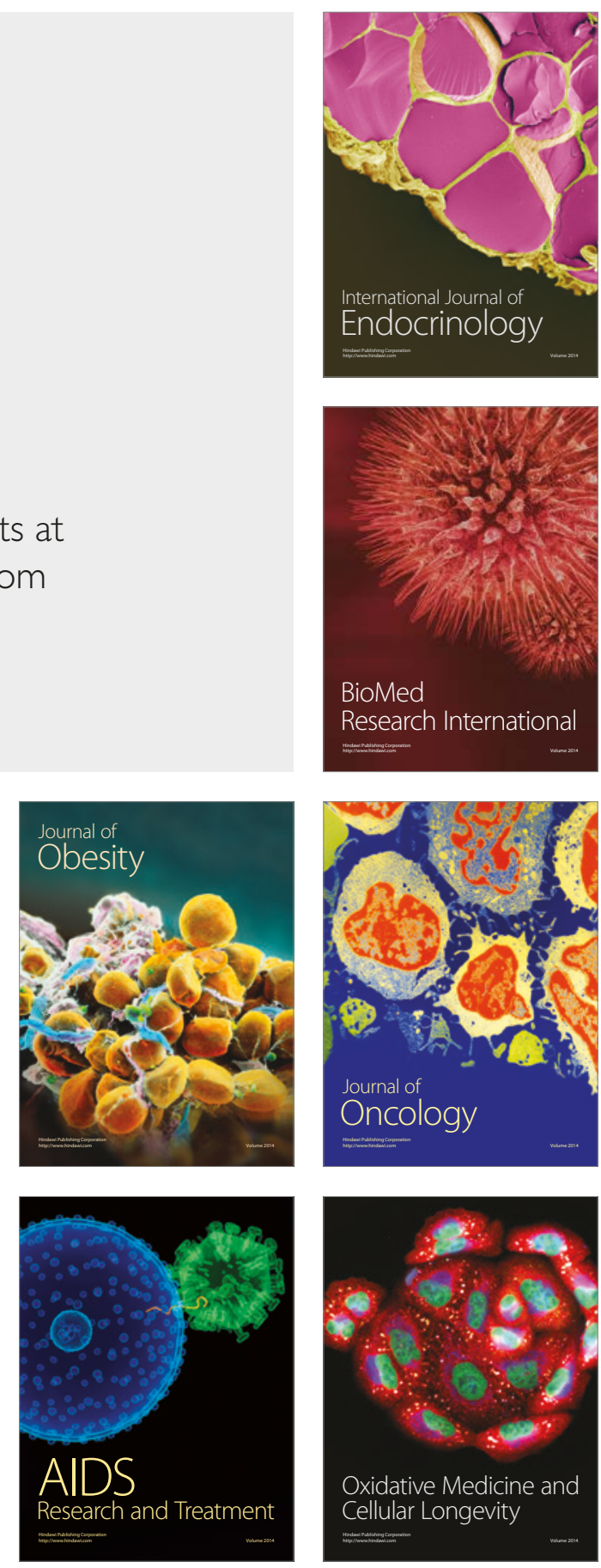